Discrete Comput Geom 38:189-200 (2007)

DOI: $10.1007 / \mathrm{s} 00454-007-1333-8$

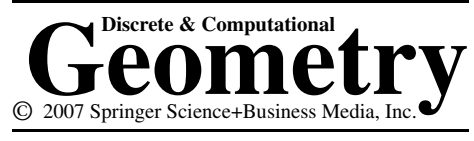

\title{
On a Generalization of Tarski's Plank Problem*
}

\author{
András Bezdek \\ Department of Mathematics, Auburn University, \\ Auburn, AL 36849-5310, USA \\ and \\ Rényi Institute of Mathematics, Hungarian Academy of Sciences, \\ Budapest H-1053, Hungary \\ bezdean@auburn.edu
}

\begin{abstract}
Answering a question of the author and K. Bezdek we prove that for any decomposition of the disk $B^{2}$ of unit radius into finite many convex cells the sum of the diameters of the incircles of the cells is at least 2. This strengthens Tarski's plank problem, where it was shown that the sum of the minimal widths of the cells is at least 2.
\end{abstract}

\section{Introduction and Preliminaries}

A strip or a plank in $E^{2}$ is a closed set bounded by two parallel lines. The distance between these lines is called the width of the strip. The minimal width (in short width) of a convex set $C$ is the minimum of the widths of the strips containing $C$.

The following theorem was conjectured by Tarski [4] and was proved by Bang [1]: If a closed convex set $C$ in $E^{2}$ is covered by a finite number of strips, then the sum of their widths is greater than or equal to the width of $C$. If $C$ is the unit disk, then there is an extremely simple proof. We repeat this proof here, mainly because our main result (Theorem 1) is a generalization of this argument:

Assume that the unit disk in $E^{2}$ is covered by strips of widths $w_{1}, \ldots, w_{n}$. Now consider the positive hemisphere in $E^{3}$ raised over the given unit disk and project perpendicularly each strip to this hemisphere. The surface area of the projection of the $i$ th strip is $\pi w_{i}$ (this is a well-known fact from calculus, already discovered by Archimedes). Since the projections cover the hemisphere, we have $\sum_{1}^{n} w_{i} \pi \geq 2 \pi$.

\footnotetext{
* The author was partially supported by the Hungarian National Science Foundation, Grant Numbers T038397 and T043520.
} 


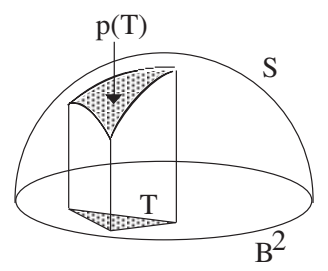

Theorem 1

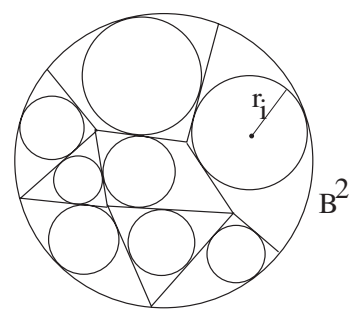

Corollary 1

Fig. 1. Theorem 1 and Corollary 1.

With K. Bezdek [2]—while working on a related plank problem called Conway's fried potato problem-we took a closer look at the above calculus fact and stated Theorem 1 as a conjectured generalization. In 1996, independently, we handled some special cases and realized that the complete solution immediately would imply Corollary 1 . Let $B^{2}$ denote the closed circular disk of radius 1 . Let $S$ be the closed hemisphere raised over $B^{2}$.

Theorem 1. If $T$ is a triangle or a strip in the plane, and $r$ is the inradius of the common part of $T$ and $B^{2}$, then the vertical projection of $T \cap B^{2}$ to the hemisphere $S$ has an area less than or equal to $2 \pi r$ (Fig. 1), where equality holds iff $T$ is a strip of width $2 r$ crossing the disk $B^{2}$.

Corollary 1 (Generalization of Tarski's Theorem). Consider a partition of disk $B^{2}$ into $n$ convex cells (Fig. 1). If $r_{1}, \ldots, r_{n}$ are the inradii of the convex cells, then $\sum_{1}^{n} r_{i} \geq 1$.

Proof. Label the cells by $C_{1}, \ldots, C_{n}$. Since $C_{1}, \ldots, C_{n}$ tile the unit disk $B^{2}$, the vertical projections $p\left(C_{1}\right), \ldots, p\left(C_{n}\right)$ tile $S$. In view of Theorem 1, their total area (which is $2 \pi$ ) has the upper bound $\sum_{1}^{n} 2 r_{i} \pi$, from which $\sum_{1}^{n} r_{i} \geq 1$ follows.

Remark 1. The inequality of Theorem 1 remains true if we replace "triangle $T$ " by "any convex region $C$ ".

Proof. Let $K$ be the incircle of $C \cap B^{2}$. It is easy to see that among the contact points of $K$ and $b d\left(C \cap B^{2}\right)$ one can find three points forming an acute triangle or two antipodal points. In both cases, the tangent lines of $K$ at these points enclose a region which has larger perpendicular projection than the convex region $C$.

Corollary 1 is stronger than Tarski's plank problem. To see this, cover each cell by the narrowest strip and notice that the widths of these strips are greater than the diameters of the incircles.

A joint paper of the author and K. Bezdek [2] on the solution of Conway's fried potato problem (Conway starts with a convex "potato" of width 1 , makes $n-1$ plane 
cuts, cutting only one piece at a time, and claims that at least one of the pieces contains a ball of diameter $1 / n$ ) solves the above convex partition problem in case of Conway-type decompositions. Further references on "plank problems" can be traced through the book of Brass et al. [3].

A complete proof of Theorem 1 was found by the author and independently by G. Kertész (unpublished). The main purpose of this paper is to prove Theorem 1.

Outline of the proof of Theorem 1. In order to simplify the description of our proof, we assign to each triangle $T$ the ratio

$$
\lambda(T)=\frac{\text { the surface area of the vertical projection of } T \cap B^{2} \text { to } S}{\text { the inradius of } T \cap B^{2}} .
$$

Note that Theorem 1 is about maximizing ratio $\lambda(T)$. In the rest of the paper, $p(\cdot)$ denotes the vertical projection from $B^{2}$ to $S, r(\cdot)$ denotes the inradius of a given convex region, while area $(\cdot)$ denotes the area (surface area resp.) of a region (of a spherical region resp.). Using this notation

$$
\lambda(T)=\frac{\operatorname{area}\left(p\left(T \cap B^{2}\right)\right)}{r\left(T \cap B^{2}\right)} .
$$

Theorem 1 claims that the maximum of $\lambda(T)$ is $2 \pi$, with equality only if $T$ is a strip crossing the disk $B^{2}$. We prove this theorem without any direct surface area computation. In Section 2 we show six elementary ways to change triangle $T$ so that ratio $\lambda(T)$ increases. In Section 3 we distinguish four types of triangles (according to how many vertices of triangle $T$ lie outside of the closed disk $B^{2}$ ) and explain how to combine the methods of Section 2 with some additional arguments to prove that strips maximize $\lambda(T)$.

\section{Techniques to Increase the Ratio of the Area of Vertical Projection and the Inradius of $T$}

First we state (without proofs) two simple elementary geometric facts:

Remark 2 (A Chord Length Inequality). Suppose a circle $S$ contains another nonconcentric circle $K$. Among all chords of $S$ tangent to $K$ both the longest and the shortest ones are perpendicular to the line connecting the centers of the circles. Furthermore, rolling the longest chord around circle $K$ gradually decreases its length.

Remark 3 (An Inradii Inequality). Let $A B C$ and $A^{*} B^{*} C$ be two homothetic isosceles triangles so that $A^{*} C=B^{*} C \leq B C=A C$. Let us shift vertex $C$ parallel to $A B$ away from the symmetry axis of $A B$ and change the positions of vertices $A$ and $B$ on the line $A B$ so that the sides $A C$ and $B C$ keep passing through the fixed points $A^{*}$ and $B^{*}$. Then the inradius of the triangle $A B C$ decreases. In particular, this is true when $A=A^{*}$, i.e. when only vertex $C$ is moving.

The following six lemmas are about different types of triangles. In each lemma we describe a continuous change of the given triangle $T$ and argue that $\lambda(\cdot)$ is increasing. 


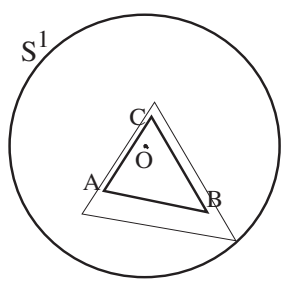

Lemma 1

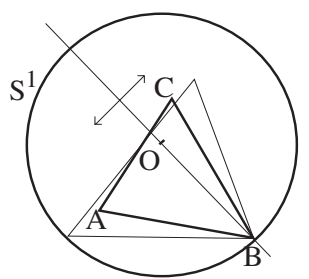

Lemma 2

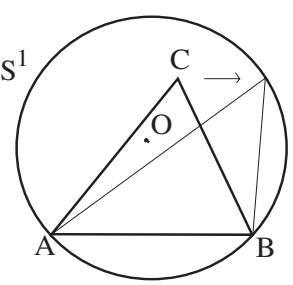

Lemma 3

Fig. 2. Lemmas 1-3.

In the rest of this section, $A, B$ and $C$ denote the vertices of triangle $T, O$ denotes the center of disk $B^{2}$ and $S^{1}$ denotes the boundary circle of disk $B^{2}$.

Lemma 1. If triangle $T$ is contained in the interior of disk $B^{2}$ (Fig. 2), then the homothetic enlargement of $T$ from the center $O$ (assuming that the image of $T$ stays in the disk $B^{2}$ ) increases $\lambda(T)$.

Proof. Assume that a very fine grid is laid over triangle $T$. Let us enlarge $T$ together with the grid by a factor $\mu>1$ so that the enlarged triangle $T^{\prime}$ is still contained in $B^{2}$. Notice that each grid cell moves farther away from $O$ and its area increases by a factor of $\mu^{2}$, thus $\mu^{2}$ area $(p(T))<\operatorname{area}\left(p\left(T^{\prime}\right)\right)$. Since $\mu r(T)=r\left(T^{\prime}\right)$, we have that $\mu(\operatorname{area}(p(T)) / r(T))<\operatorname{area}\left(p\left(T^{\prime}\right)\right) / r\left(T^{\prime}\right)$, which means that $\lambda(T)$ increases.

Lemma 2. Assume that triangle $T$ is contained in disk $B^{2}$ so that exactly one vertex, say $B$, belongs to the boundary circle $S^{1}$ (Fig. 2). Then the perpendicular affinity whose axis is $O B$ and which changes triangle $T$ to a triangle $T^{\prime}$, which lies in disk $B^{2}$ and has a second vertex on $S^{1}$, increases the assigned ratio $\lambda(T)$.

Proof. Assume that a very fine grid is laid over triangle $T$. Let us apply a perpendicular affinity of a factor $\mu>1$ to $T$ and to the grid so that the enlarged triangle $T^{\prime}$ is still contained in $B^{2}$. Each grid cell moves away from $O$ and its area increases by a factor of $\mu$, thus $\mu$ area $(p(T))<\operatorname{area}\left(p\left(T^{\prime}\right)\right)$. Consider now the inverse of this affinity (i.e. the one with factor $1 / \mu$ ). The image of the incircle of $T^{\prime}$ is an ellipse of half diameters $r\left(T^{\prime}\right)$ and $(1 / \mu) r\left(T^{\prime}\right)$, thus $r(T)>(1 / \mu) r\left(T^{\prime}\right)$. Dividing the previous inequality with the latter one, we have that $\operatorname{area}(p(T)) / r(T)<\operatorname{area}\left(p\left(T^{\prime}\right)\right) / r\left(T^{\prime}\right)$, which means that $\lambda(T)$ increases.

Lemma 3. Assume that triangle $T$ is contained in disk $B^{2}$ with exactly two vertices, say with $A$ and $B$, belonging to the boundary circle $S^{1}$ (Fig. 2). Then as one moves the third vertex $C$ of $T$ parallel to $A B$, away from the center $O$, while keeping the other two vertices fixed, the ratio $\lambda(T)$ increases.

Proof. This time, instead of using a fine grid, let us subdivide $T$ using lines parallel to $A B$ into very thin trapezoids. As we move $C$ parallel to $A B$, we essentially apply 

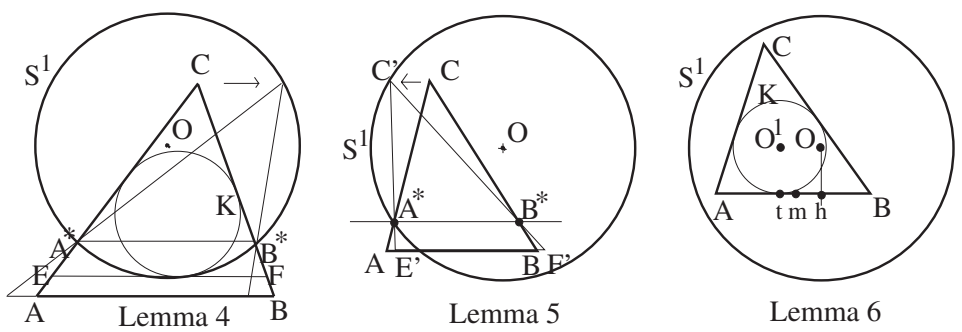

Fig. 3. Lemmas 4-6.

an affine transformation to each trapezoid preserving both their length and width. Since $C$ moves away from the center $O$, the area of the projection of each trapezoid to the hemisphere increases. Thus, area $(p(T))<\operatorname{area}\left(p\left(T^{\prime}\right)\right)$. According to Remark 3 , the inradius decreases, thus $r(T)>r\left(T^{\prime}\right)$. Dividing these two inequalities with each other, we get that area $(p(T)) / r(T)<\operatorname{area}\left(p\left(T^{\prime}\right)\right) / r\left(T^{\prime}\right)$, which means that $\lambda(T)$ increases.

Lemma 4. Assume that only one of the vertices of triangle $T$, say $C$, belongs to the interior of disk $B^{2}$ and assume that side $A B$ is disjoint from $B^{2}$ (Fig. 3). Let $A^{*}\left(B^{*}\right.$ resp.) be the point where the side $A C$ ( $B C$ resp.) intersects the boundary circle $S^{1}$. Also assume that side $A B$ is disjoint from $B^{2}$ and is parallel to the segment $A^{*} B^{*}$. If triangle $T$ is changed so that (i) the vertex $C$ moves parallel to $A^{*} B^{*}$ away from the center $O$, (ii) the side $A C$ ( $B C$ resp.) keeps passing through $A^{*}$ ( $B^{*}$ resp.) and (iii) the line of $A B$ remains unchanged, then $\lambda(T)$ increases.

Proof. Let us denote a new position of triangle $T$ by $T^{\prime}$. The area argument used in the previous lemma immediately gives area $\left(p\left(T \cap B^{2}\right)\right)<\operatorname{area}\left(p\left(T^{\prime} \cap B^{2}\right)\right)$. Now we show that $r\left(T \cap B^{2}\right)>r\left(T^{\prime} \cap B^{2}\right)$, which, as in the proof of the previous lemma, implies that $\lambda(T)$ increases. Choose triangle $E F C$ so that $E F$ is parallel to $A B$ and $E F C$ and $T \cap B^{2}$ have the same incircle. Let us move $C$ according to Lemma 4 and find out how the inradius of $T \cap B^{2}$ changes. It is easy to see that the incircle rolls on $S^{1}$ to the right and thus cannot intersect the line $E F$ and therefore it is smaller than the incircle of triangle $T$. However, the latter, in view of Remark 3, is smaller than the incircle of $T$.

Lemma 5. Assume that exactly two of the vertices of triangle $T$, say $B$ and $C$, belong to the interior of the disk $B^{2}$ and assume $B C \geq A C$ (Fig. 3). Let $A^{*}$ be the common point of the side $A C$ and the boundary circle $S^{1}$. Let $B^{*}$ be a point on the side $B C$ so that $A B$ is parallel to $A^{*} B^{*}$. If triangle $T$ is changed so that (i) the vertex $C$ moves parallel to $A^{*} B^{*}$ away from the center $O$, (ii) the side $A C$ ( $B C$ resp.) keeps passing through $A^{*}\left(B^{*}\right.$ resp.) and (iii) sideline $A B$ remains the same, then $\lambda(T)$ increases.

Proof. Let us denote a new position of triangle $T$ by $T^{\prime}$. The area argument used in the proof of Lemma 3 gives area $\left(p\left(T \cap B^{2}\right)\right)<\operatorname{area}\left(p\left(T^{\prime} \cap B^{2}\right)\right)$. In view of Remark 3 we have $r\left(T \cap B^{2}\right)>r\left(T^{\prime} \cap B^{2}\right)$. Dividing the area inequality with the inradius 
inequality we get that area $(p(T)) / r(T)<\operatorname{area}\left(p\left(T^{\prime}\right)\right) / r\left(T^{\prime}\right)$, which means that $\lambda(T)$ increases.

Many extreme value problems are solved by local improvements. For example, when finding the smallest area triangle circumscribed to a given circle, one tries to roll one of the sides along the circle, keeping the other two fixed. It is easy to see that the area of the triangle can be decreased this way, unless the contact points are midpoints of the edges of the triangle. The following lemma shows that such improvements, in case of our problem, work only under very special circumstances.

Lemma 6. Assume that vertex $B$ of triangle $T$ belongs to the interior of disk $B^{2}$. Also assume that side $A B$ touches the incircle $K$ of $T \cap B^{2}$. Let $m$ be the midpoint of the segment $A B \cap B^{2}$, let $t$ be the tangency point of the side $A B$ and the incircle of $T \cap B^{2}$ and let $h$ be the midpoint of the chord of $B^{2}$ containing the side BC (Fig. 3). If the order of these three points is different from $t-m-h$ (here $t$ and $h$ might coincide with $m$ ), then rolling the sideline $A B$ around the incircle $K$ in one of the two directions increases the ratio $\lambda(T)$.

Proof. Without loss of generality, assume that $A B$ is horizontal and $A$ is the left endpoint. We prove Lemma 6 indirectly. If $A$ belongs to $B^{2}$, then the indirect assumption (without loss of generality) means that among the points $m, h$ and $t$, point $m$ is the most left and neither $t$ nor $h$ coincides with it. If $A$ does not belong to $B^{2}$, then $m$ has to be left from $h$ and thus the indirect assumption means again that among the points $m, h$ and $t$, point $m$ is the most left and neither $t$ nor $h$ coincides with it. We distinguish two cases (Fig. 4):

(A) In the triplet $\{m, h, t\}, t$ is the second point from the left and it is different from $h$.

(B) In the triplet $\{m, h, t\}, h$ is the second point from the left and it might coincide with $t$.

In both cases rolling the sideline $A B$ counterclockwise along the incircle of $T$ increases $\lambda(T)$. As Fig. 4 illustrates, the reasons are different in cases (A) and (B). It turns out that the small triangle $T^{\prime}$ (which represents the area of loss) can be placed in the tri-

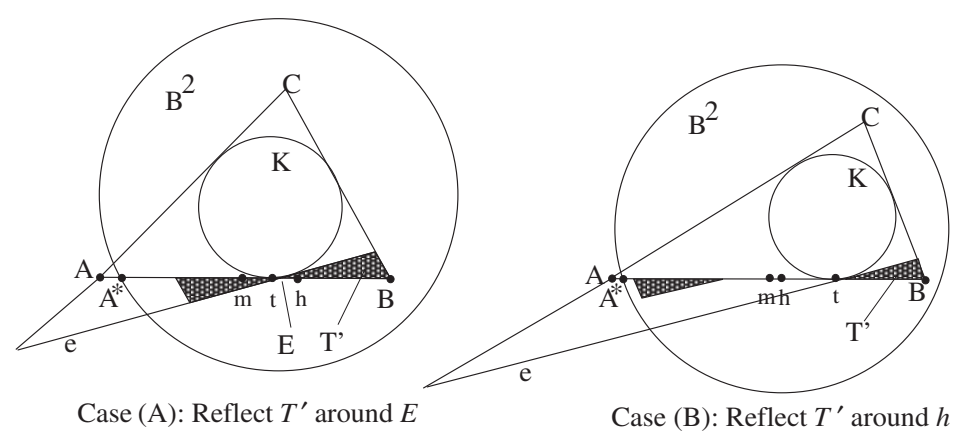

Fig. 4. Lemma 6 . 
angle representing the area of gain so that the corresponding points of these triangles get farther away from $O$ and thus the area of the vertical projection of $T$ to the hemisphere $S$ increases.

\section{Proof of Theorem 1}

A simple compactness argument shows that there exists a triangle $T$ or a strip $T$ for which $\lambda(T)$ is maximal. We show that the maximum is not achieved in the case of triangles. We distinguish four types of triangles according to how many of their vertices lie outside of $B^{2}$ (see Fig. 5).

Proposition 1. If triangle $T$ is a subset of $B^{2}$ (see Case 0 in Fig. 5), then it cannot maximize $\lambda(T)$.

Proof. Using Lemmas 1-3 we conclude that when maximizing $\lambda(T)$ we may assume that all three vertices $A, B$ and $C$ of $T$ lie on the boundary circle of $B^{2}$. Let $P, Q$ and $R$ be the points where the incircle of $T$ is touching the sides $A C, C B$ and $B A$ (Fig. 6). Let $O^{1}$ be the center of the incircle $K$ of $T$. Without loss of generality, we may assume that ray $O O^{1}$ intersects the shorter circular arc $P Q$. Let $e$ be the other support line of $K$ which is parallel to $A B$. Line $e$ has to touch $K$ also on the shorter circular arc $P Q$, say, at $R^{*}$. Without loss of generality we may assume that $P$ and $R^{*}$ are not separated by line $O O^{1}$. In view of Remark 2, we know that line $e$ cuts off less from disk $B^{2}$ than chord $A C$ and thus a strip of width $2 r$ has larger $\lambda(\cdot)$.

Proposition 2. If triangle $T$ has exactly two of its vertices of $T$ outside of $B^{2}$ (see Case 1 in Fig. 5), then it cannot maximize $\lambda(T)$.

Proof. First we consider a special case:

Lemma 7. Triangle $T$ with vertices $A, B$ and $C$ cannot maximize $\lambda(T)$ if vertex $C$ lies on the boundary of $B^{2}$ and side $A B$ is disjoint from $B^{2}$.

Proof. Let $K$ be the incircle of $T \cap B^{2}$ with center $O$ (Fig. 7). It is enough to prove Lemma 7 if circle $K$ is tangent to sides $C A, C B$ and the boundary circle of $B^{2}$. De-

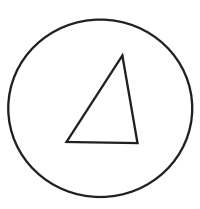

Case 0

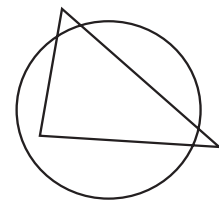

Case 1

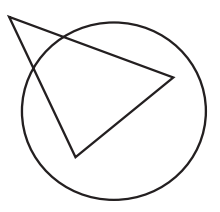

Case 2

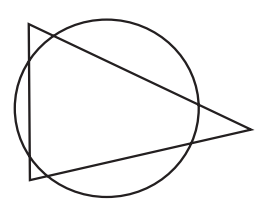

Case 3

Fig. 5. Four types of triangles. 


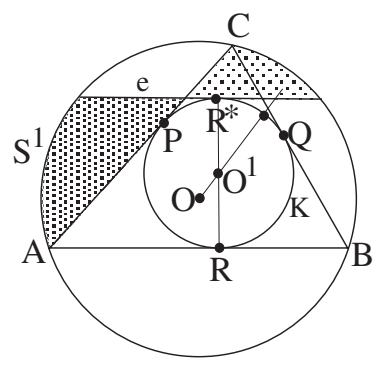

Fig. 6. Proposition 1 .

note the three contact points (in the above order) by $P, Q$ and $R$. Ray $R O$ intersects $K$ between $P$ and $Q$, say, at $R^{\prime}$. Similarly, ray $Q O$ intersects $K$ between $P$ and $R$, say, at $Q^{\prime}$. According to Remark 2, among all chords of $B^{2}$ which are tangent to circle $K$, the one through $R^{\prime}$ is the longest. The order of the points $R^{\prime}, P$ and $Q^{\prime}$ along circle $K$ implies that the chord through $P$ is longer than the one through $Q^{\prime}$. The monotonicity of the area cut off from $K$ by chords of decreasing length implies our inequality.

Now we consider the general case of Proposition 2. Let $K$ be the incircle of $T \cap B^{2}$ with center $O$ (Fig. 7). We may assume that $K$ is tangent to the sides $C A, C B$ and $A B$, otherwise Lemma 4 reduces this case to the special case we just discussed. Denote the three contact points (in the above order) by $P, Q$ and $R$. Ray $R O$ intersects $K$ between $P$ and $Q$, say at $R^{\prime}$. Let $x$ be the chord of $B^{2}$ which is parallel to $A B$ and touches $K$ at $R^{\prime}$. Let $X$ be the circular segment (by definition a circular segment is a region bounded by an arc and a chord) which is cut off from $B^{2}$ by the chord $x$ and contains $C$. Denote by $T^{\prime}$ a triangle which can be obtained from triangle $T$ by shifting the sideline $A B$ away from $C$ until $A B$ becomes disjoint from $B^{2}$. Let $K^{\prime}$ be the incircle of $T^{\prime} \cap B^{2}$. Let $x^{\prime}$ be the chord of $B^{2}$ which is parallel to $A B$ and touches $K^{\prime}$ and separates $K^{\prime}$ from line $A B$. Finally let $X^{\prime}$ be the circular segment which is cut off from $B^{2}$ by the chord $x^{\prime}$ and which

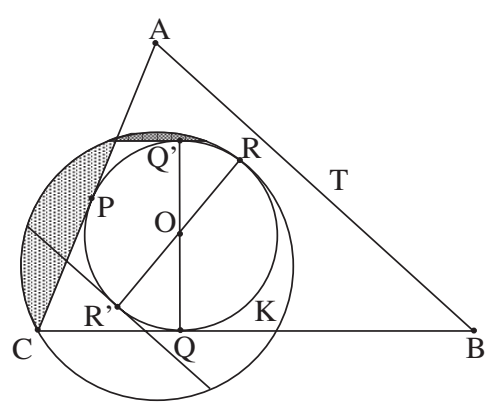

Special case (Lemma 7)

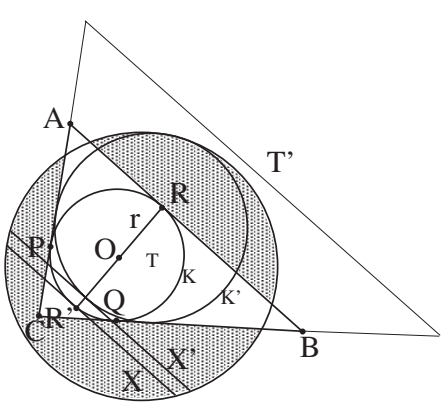

General case

Fig. 7. Proposition 2. 

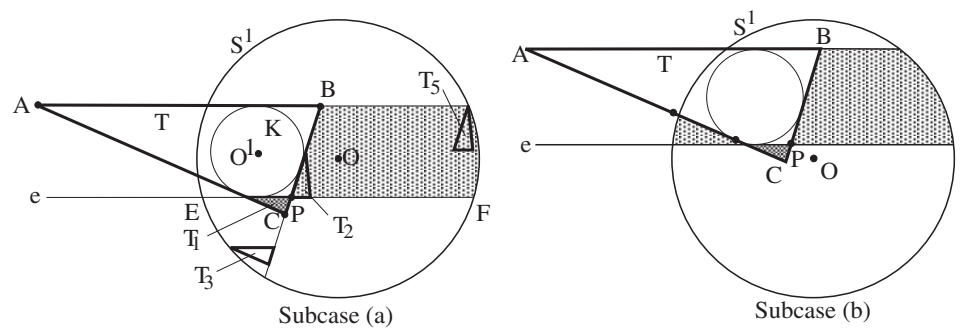

Fig. 8. Proposition 3.

separates $C$ from $K^{\prime}$. We have that

$$
\operatorname{area}(p(X))<\operatorname{area}\left(p\left(X^{\prime}\right)\right)<\operatorname{area}\left(p\left(B^{2} \backslash T^{\prime}\right)\right),
$$

where the first inequality follows from $X \subset X^{\prime}$ and where the second inequality follows from Lemma 7 applied for triangle $T^{\prime}$. Let $Y$ be that circular segment which is cut off from $B^{2}$ by line $A B$ and which does not contain $C$. Adding area $(p(Y))$ to the first and to the last terms of the above inequality chain we get that $2 \pi-2 r \pi<\operatorname{area}\left(p\left(B^{2} \backslash T\right)\right)$, which is what we had to prove.

Proposition 3. If triangle $T$ has exactly one of its vertices outside of $B^{2}$ (see Case 2 in Fig. 5), then it cannot maximize $\lambda(T)$.

Proof. Let $A$ be the vertex of $T$ which lies outside of $B^{2}$ (Fig. 8). Indirectly assume triangle $T$ maximizes $\lambda(T)$. In view of Lemma 4 we have that $B C$ is the shortest side of $T$. Without loss of generality assume $A B \geq A C$. Denote by $e$ the line which is parallel to $A B$ and is tangent to incircle $K$. Let $H$ be the strip bounded by lines $A B$ and $e$.

We show that the $\lambda(T)<\lambda(H)$, contradicting the maximal choice of $T$. Equivalently, we show that area $(p(T \backslash H))<\operatorname{area}(p(H \backslash T)$. How can we prove this without surface area computation and without changing triangle $T$ ? Let triangle $T \backslash H$ be denoted by $T_{1}$. The basic idea is to show that there is an isometry $f$ from $T_{1}$ to $H \backslash T$ (i.e. a copy of triangle $T_{1}$ can be placed in $\left.H \backslash T\right)$ so that for each point $X$ of $T_{1}$ the tangent plane of $S$ at $p(f(X))$ is steeper than the one at $p(X)$. It is just a technical complication that $f$ will be given as a composition of several isometries, where only the image of the last isometry belongs to the strip $H \backslash T$. It turns out that we achieve our goal by studying two subcases:

Subcase (a): Line e Separates the Center of $B^{2}$ from Vertex $C$. Let $E F$ be the chord $e \cap B^{2}$. Denote by $P$ the intersection point of $E F$ and $B C$. We define several congruent triangles:

Definition 1. Triangle $T_{1}$ was defined as $T \backslash H$. Let triangle $T_{2}$ be the image of $T_{1}$ under the reflection around axis $O^{1} P$, where $O^{1}$ is the incenter of $T$.

Lemma 8. Triangle $T_{2}$ is a subset of strip $H$. 


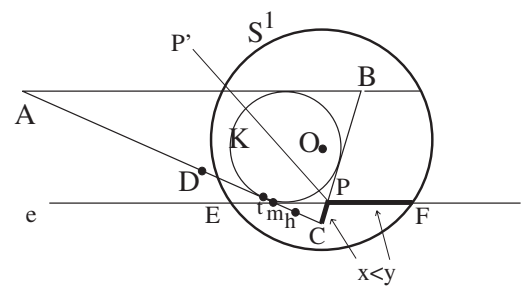

(a)

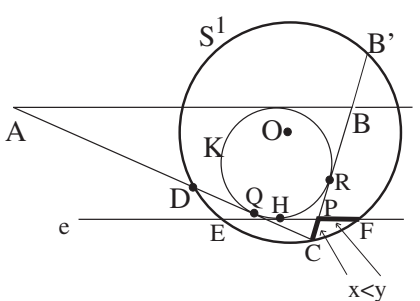

(b)

Fig. 9. Lemma 8: (a) the general case and (b) the special case.

Proof. To see that $T_{2} \subset H$ we need to verify that (i) the horizontal side of $T_{1}$ is shorter than the width of $H$, and (ii) $P C<P F$. Since $A B$ is the longest side of $T_{1}$ we have that $\angle A C B \geq \pi / 3$ and thus the horizontal side of $T_{1}$ has length at most $r \sqrt{3}$, which implies (i). It is a little bit more complicated to check that $P C<P F$. By the indirect assumption $T$ is maximizing $\lambda(T)$, thus applying Lemma 6 to the side $A C$ we get that the three critical points mentioned in the lemma are in the order of $t-m-h$ or $h-m-t$ (see Fig. 9(a)). Since the midpoint $m$ of $A C \cap B^{2}$ separates $A$ from the chord's midpoint $h$, the order must be $t-m-h$. Thus, reflecting $C$ around the contact point $t$ we get a point $D$ outside of the disk $B^{2}$. We want to prove the inequality $C P<P F$. Therefore, we can reduce the problem to special cases by repositioning the disk $B^{2}$ so that along the motion (i) $P F$ decreases, (ii) center $O$ stays above the line $e$, (iii) $D$ stays outside of disk $B^{2}$ and (iv) vertices $C$ and $B$ remain contained by disk $B^{2}$. It is easy to see that simple compositions of shifts parallel to $A C$ toward $A$ and rotations around $C$ and $B$ (if necessary) will reduce the problem to the one when both $D$ and $C$ belong to the boundary of $B^{2}$ (see Fig. 9(b)). In this case chord $D C$ is shorter than chord $e \cap B^{2}$. Thus $C R=Q C<H F$. Subtracting the equation $P R=P H$ from this inequality we get that $C P<P F$, which is what we wanted.

Let us return to subcase (a) depicted in Fig. 8. Let $P O^{1}$ be the bisector of the angular sector $E P B$. If the center $O$ of $B^{2}$ belongs to the angular sector $E P P^{\prime}$, then it is obvious that area $\left(p\left(T_{1}\right)\right)<\operatorname{area}\left(p\left(T_{2}\right)\right)$, which implies Proposition 3. In the rest of the argument we assume that center $O$ of $B^{2}$ belongs to the angular sector $P^{\prime} P F$.

Definition 2. Let triangle $T_{3}$ be the one which we get by translating $T_{1}$ along the line $B C$ away from $O$ until it reaches the boundary of disk $B^{2}$ (see Fig. 8: subcase (a)).

Since $\angle E P B>\pi / 2$, the line which is perpendicular to $B C$ and passes through $P$ is separating the center of $B^{2}$ from triangle $T_{1}$, thus we have area $\left(p\left(T_{1}\right)\right)<\operatorname{area}\left(p\left(T_{3}\right)\right)$.

If the contact point of $T_{3}$ and $S^{1}$ belongs to the horizontal side of $T_{3}$, then a rotation which takes each point of the triangle away from $O$ is used until a second vertex belongs to $S^{1}$. Denote by $T_{4}$ this new triangle ( $T_{4}$ is not shown in Fig. 8). It is easy to see that $\operatorname{area}\left(p\left(T_{3}\right)\right)<\operatorname{area}\left(p\left(T_{4}\right)\right)$. If the contact point of $T_{3}$ and $S^{1}$ does not belong to the horizontal side of $T_{3}$, then a counterclockwise rotation is used until a second vertex belongs to $S^{1}$. We also denote by $T_{4}$ this new triangle. Let us remind ourselves that $T_{3}$ is 


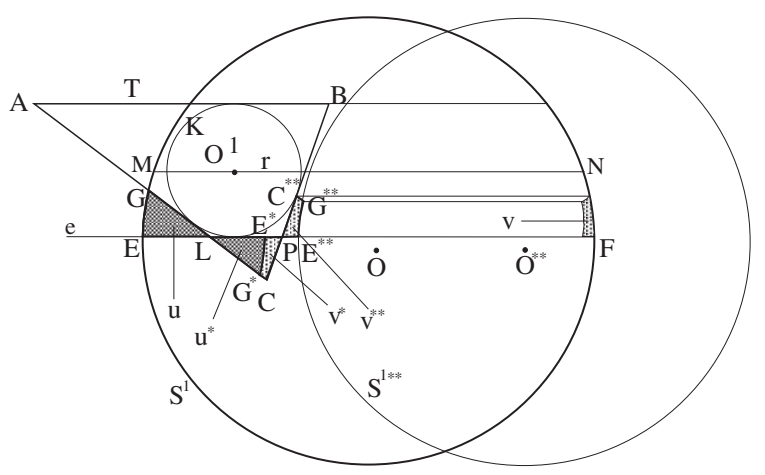

Fig. 10. Subcase (b) of Proposition 3.

similar to $A B C$, where $A C \leq C B$. A simple analysis of the intersection of $T_{3}$ and thin annuli centered at the center of rotation reveals that again area $\left(p\left(T_{3}\right)\right)<\operatorname{area}\left(p\left(T_{4}\right)\right)$.

Definition 3. Consider that translate of $T_{2}$ which lies in the region $H \backslash T$ and touches the boundary of $H \backslash T$ either at its right-upper or right-lower corner (it is easy to see that at least one corner can be contacted). If it touches the right-upper corner we call it $T_{5}$. If it touches the right-lower corner we call it $T_{6}$.

It is easy to see that both $T_{5}$ and $T_{6}$ can be rotated around their contact points so that they remain contained in $H$ and have another vertex on the boundary of $B^{2}$. Since the new triangles have the same relative positions with respect to $B^{2}$ as triangles $T_{4}$, we can conclude that area $\left(p\left(T_{1}\right)\right) \leq \operatorname{area}\left(p\left(T_{3}\right)\right)=\operatorname{area}\left(p\left(T_{5}\right)\right)=\operatorname{area}\left(p\left(T_{6}\right)\right)$, which is what we wanted.

Subcase (b): Line e Does Not Separate the Center of $B^{2}$ from Vertex $C$. In the rest of the proof we use Fig. 10, where we continue using the notation of Figs. 8 and 9. Let $H$ again be the strip which is parallel to $A B$ and contains the incircle of $T$. Recall that $T_{1}$ denotes the triangle $T \backslash H$. We show that area $\left(p\left(T_{1}\right)\right)<\operatorname{area}(p(H \backslash T))$.

We need to introduce a couple of new points and regions. Let $L$ be the intersection of lines $e$ and $A C$. Denote by $u$ the region enclosed by $A C, e$ and $S^{1}$. Let $u^{*}$ be the common part of $T_{1}$ and the image of $u$ under the reflection around $L$. Let $v^{*}$ be the region $T_{1} \backslash u^{*}$. Let $v^{* *}$ be the image of $v^{*}$ under the reflection around $E$. Next we show that $v^{*} \subset H$.

To see this, it is enough to verify that $C^{* *} \in H$ and $G^{* *} \in H$. The first inclusion follows from the assumption that $B C$ is the shortest side of $T$. Indeed, this implies that $\angle A C B \geq \pi / 3$ and thus $P C^{* *}=C P<\sqrt{3} r<2 r$, which means that $C^{* *} \in H$. It is harder to see the second inclusion. The above angle inequality implies again that $E E^{* *}=2 L P<2 \sqrt{3}$. The composition of the reflections around $L$ and then around $P$ take the circle $S^{1}$ to $S^{1 * *}$. Notice that the second circle is obtained from $S^{1}$ by a horizontal translation of length $<2 \sqrt{3} r$. Simple calculus shows that $M N \leq 2 \sqrt{3} r$, thus $M N$ and $S^{1 * *}$ intersect each other in the interior of $B^{2} . \angle A C B \geq \pi / 3$ implies that distance between $G^{*}$ and $e$ is at most $r$ and then the same applies for $G^{* *}$, i.e. we have the desired inclusion $G^{* *} \in H$. 
Finally change the region $v^{* *}$ into region $v$ using the following transformation, called "shift to right": Treat region $v^{* *}$ as a union of horizontal segments and translate each segment horizontally to the right until the right endpoint belongs to $S^{1}$. Every point of $u$, when reflected around $L$ gets closer to the center $O$ of $B^{2}$. Similarly, every point of $v^{*}$, when reflected around $E$ and then "shifted to right", moves away from the center $O$ of $B^{2}$. The last two observations imply that area $\left(p\left(T_{1}\right)\right)<\operatorname{area}(p(u \cup v))<\operatorname{area}(p(H \backslash T))$, which is what we wanted to prove.

Proposition 4. A triangle $T$, with all three vertices outside of disk $B^{2}$ (see Case 3 in Fig. 5), cannot maximize $\lambda(T)$.

Proof. It is easy to see that rolling one of the sides improves the area of the projection and thus $T$ cannot be optimal.

\section{References}

1. K. Bang, A solution of the "plank problem", Proc. Amer. Math. Soc. 2 (1951), 990-993.

2. A. Bezdek and K. Bezdek, A solution of Conway's fried potato problem, Bull. London. Math. Soc. 27 (1995), 492-496.

3. P. Brass, W. Moser and J. Pach, Research Problems in Discrete Geometry, Springer-Verlag, New York, 2005.

4. A. Tarski, Further remarks about the degree of equivalence of polygons, Parametr 2 (1932), 310-314.

Received March 4, 2006, and in revised form May 5, 2006. Online publication July 13, 2007. 